\title{
CATEGORÍA SOCIAL Y ETNICIDAD EN FRANCIA
}

\section{EGUZKI URTEAGA}

Departamento de Sociología I. Escuela Universitaria de Trabajo Social. Universidad del País Vasco Vitoria. Centro de Investigación IKER (laboratorio asociado al CNRS francés).

\section{RESUMEN}

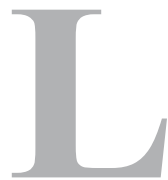

as investigaciones sociológicas llevadas a cabo en Francia en materia de inmigración tienden a asociar de manera creciente la categoría social y la etnicidad en torno a cuatro objetos y problemáticas: 1) las manifestaciones del racismo y de la discriminación en el trabajo, que dificultan el acceso de los inmigrantes al empleo y, más aún, a los altos cargos, 2) la segregación urbana con el aislamiento y la concentración de la población inmigrante en ciudades y barrios determinados, que son ampliamente estigmatizados tanto por las autoridades como por los medios de comunicación, lo que dificulta su integración, 3) la descalificación y la etnicización creciente del sistema educativo en detrimento de un planteamiento en términos de situación social y de capital cultural, y 4) los disturbios urbanos que se reproducen como consecuencia de la discriminación y de la fragilización de la población inmigrante y tras unos incidentes con la policía.

Palabras clave: categoría social, etnicidad, discriminación, racismo, segregación, Francia.

\begin{abstract}
Sociological research in France on the subject of immigration tends to group social category and ethnicity together in terms of four issues and problems: 1) manifestations of racism and discrimination in the workplace, which hinder immigrants' access to employment and particularly to positions of responsibility, 2) urban segregation, which includes isolation and the concentration of immigrant populations in certain cities and areas that are widely stigmatized by the authorities and the mass media alike, making integration difficult, 3) the disqualification and growing ethnicisation of the educational system to the detriment of an approach based on social situation and cultural capital, and 4) urban unrest, which occurs as a consequence of the discrimination and increased fragility of the immigrant population, and following incidents with the police.
\end{abstract}

Key words: social category, ethnicity, discrimination, racism, segregation, France. 


\section{INTRODUCCIÓN}

En treinta años, los trabajos sobre la inmigración se han multiplicado, ya que numerosos campos de la sociología (urbana, de la educación, del empleo, de la vida política, de las religiones) se han cruzado con ella para medir su impacto social. Los objetos, conceptos y métodos han evolucionado y las categorías de la sociología de la inmigración se han ilustrado en varias investigaciones empíricas. El estudio comparativo llevado a cabo sobre la instalación de los inmigrantes ha conducido a Castels (1984) a ver en el racismo y en la discriminación racial el origen de la formación de las minorías étnicas en Europa. Después de mostrar cierta reticencia a la hora de reconocer las discriminaciones, Francia ha aceptado, en los ámbitos tanto político como científico, hacerlo para demostrar que el éxito de la integración supone la desaparición de todas las discriminaciones. "Completamente desfasado con respecto a la realidad que pretendía describir, el lenguaje se convertía no tanto en un instrumento de análisis sino en lo que lo imposibilitaba".

En este sentido, las investigaciones sociológicas llevadas a cabo en Francia ${ }^{2}$ en materia de inmigración tienden a asociar de manera creciente la clase social y la etnicidad en torno a cuatro objetos y problemáticas: 1) las manifestaciones del racismo y de la discriminación en el trabajo, que dificultan el acceso de los inmigrantes al empleo y, más aún, a los altos cargos, 2) la segregación urbana con el aislamiento y la concentración de la población inmigrante en ciudades y barrios determinados que son ampliamente estigmatizados tanto por las autoridades como por los medios de comunicación, 3) la descalificación y la etnicización creciente del sistema educativo en detrimento de un planteamiento en términos de situación social y de capital cultural, y 4) los disturbios urbanos ${ }^{3}$ que se reproducen como consecuencia de la discriminación y de la fragilización de la población inmigrante y tras unos incidentes con la policía.

\section{EL RACISMO Y LA DISCRIMINACIÓN EN EL TRABAJO}

La naturaleza misma de la empresa y del empleo cambia a partir de los años 1980. El desempleo masivo se instala de manera duradera e impide el acceso al empleo, especialmente de la juventud popular, y, en su seno, de la parte proveniente de la inmigración. El acceso al trabajo estable se produce después de un largo periodo de precariedad laboral donde las prácticas, los contratos definidos y la interinidad se encadenan. Esta situación aleja a los jóvenes de los sindicatos porque no pertenecen a las empresas o no se identifican con las centrales. A partir de los años 1990, la estigmatización de los jóvenes provenientes de la inmigración se produce a través de los testimonios individuales en la prensa y se manifiesta por mediación de la discriminación en la contratación. Afecta en primer lugar a los jóvenes que acumulan los estigmas: hijos de inmigrantes, de musulmanes, de obreros y de los suburbios urbanos.

Si Bataille (1997) es uno de los primeros en estudiar el racismo en el ámbito laboral, este tema ha sido tabú durante un largo periodo, como lo atestigua el título del artículo "Racismo y discriminación en el trabajo: una realidad ocultada" . Se sitúa en la continuidad de una investigación europea que establece que Francia no dispone de ningún instru-

1 FASSIN, D., «L'invention française de la discrimination», Revue française de science politique, vol.52, n4, 2002, p. 214.

2 WIEVIORKA. M., La France raciste. Paris, Seuil, 1992.

TAGUIEFF, P-A., Face au racisme. Paris, La Découverte, 1991.

3 AVENEL, C., Sociologie des quartiers sensibles. Paris, Armand Colin, 2005.

4 DE RUDDER, V.; VOURC'H, F.; TRIPIER. M., "Racisme et discrimination dans le travail: une réalité occultée", L'Homme et la société, n 121-122, 1996. 
mento de medida de la discriminación y que su denegación es frecuente ${ }^{5}$. Denunciando la ley del silencio en torno a las discriminaciones racistas en la empresa, ciertos investigadores han expuesto los procedimientos directos e indirectos así como los argumentos de los empleadores que desean evitar a los "indeseables". Muestran que las leyes de la República dejan a los empleadores numerosas vías de no respetarlas cuando lo consideran oportuno, teniendo en cuenta los procedimientos de contratación y la ausencia de un movimiento de opinión que deslegitima tales prácticas ${ }^{6}$. La discriminación en la contratación explica, en parte, el auge del empresariado étnico entre las nuevas generaciones ${ }^{7} . ¿^{\text {Este }}$ acceso desigual al empleo, incluso precario, es el fruto de un cúmulo de deficiencias o la expresión de un racismo latente? En realidad, las carencias y las discriminaciones se compaginan para conducir a dicho resultado ${ }^{8}$.

Una encuesta observa la presencia de las nuevas generaciones de inmigrantes en un mayor número de sectores y de profesiones, pero subraya una sobre-representacion de los descendientes de inmigrantes magrebíes entre los desempleados y los precarios. La deficiencia vinculada a un origen heredado "atestigua de la existencia de unas discriminaciones cuya amplitud y persistencia relevan más del sistema en sí que de las disposiciones individuales de los empleadores". La investigación de Silberman y Fournier (2004) concluye igualmente a un "castigo étnico" que padecen los descendientes de inmigrantes magrebíes y, cada vez más, los jóvenes provenientes de África subsahariana. El interés de esta investigación estriba en la aplicación al caso francés de las teorías americanas sobre la asimilación segmentada ${ }^{9}$. Lo que aproxima la situación de los negros en Estados Unidos a la de los descendientes de la inmigración post-colonial en un contexto histórico de dominación asociado a los efectos de la escolarización diferencial y que reproduce una infravaloración duradera.

El reconocimiento de las discriminaciones por las administraciones públicas ha provocado la puesta en marcha de unos dispositivos de lucha contra estas últimas. Progresivamente, la discriminación vinculada al origen ha sido diluida en un dispositivo general que concierne tanto al sexo, a la edad o a la orientación sexual como a la deficiencia. La responsabilidad de las desigualdades que padecen sigue recaiendo en los interesados. Según Fassin, se ha pasado a la denegación, puesto que, "apenas reconocidas oficialmente, las discriminaciones raciales se ven excluidas del espacio público. Con el discurso sobre la igualdad de oportunidades, se difumina la realidad de las discriminaciones, $y$, con la instauración de dispositivos indiferenciados, se borra su carácter racial" ${ }^{10}$. Para Lorcerie $^{11}$, la lucha contra las discriminaciones se enfrenta a un "etno-nacionalismo" que impregna las mentalidades y las instituciones: "las instituciones republicanas promueven el anti-racismo y se muestran preocupadas por la igualdad. A pesar de ello, el primordialismo nacional, movilizado sistemáticamente en la vida política sin ser reconocido, sigue vigente y es legítimo para los agentes públicos".

Si la discriminación supone una relación de poder y de dominación, el racismo ordinario se refiere a la esfera de las actitudes y de las opiniones y se declina diferentemente

5 WRENCH, J.; REA, A.; OUALI. N., Migrants, Ethnic, Minorities and the Labour Market. London, Macmillan, 1999.

6 DE RUDDER, V.; POIRET, C.; VOURC'H, F., L'inégalité raciste. Paris, PUF, 2000, pp. 140-146.

7 SANTELLI, E., La mobilité sociale dans l'immigration. Toulouse, Presses Universitaires du Mirail, 2001.

8 AUBERT, F.; TRIPIER, M.; VOURC'H, F., Jeunes issus de l'immigration: de l'école à l'emploi. Paris, CIEMI/ L'Harmattan, 1997.

9 PORTES, A., "Children of immigrants: segmented assimilation and its determinants", in PORTES, A., et al, The economic sociology of immigration. New York, Sage, 1995.

10 FASSIN, D., "Du déni à la dénégation. Psychologie politique de la représentation des discriminations", in FASSIN, D., FASSIN, E., De la question sociale à la question raciale. Paris, La Découverte, 2006, p. 140.

11 LORCERIE, F., "Le primordialisme français, ses voies, ses fièvres”, in SMOUTS, M.-C., La situation post-coloniale. Paris, Presses de Sciences Po, 2007, p. 301. 
según los entornos sociales. El discurso racista en los sectores populares está generalmente relacionado con las condiciones de vida y con las representaciones que se despliegan en el trabajo y fuera de él. Ha sido objeto de varios estudios, ya que las clases media y alta se escapan de la competencia profesional y residencial con los inmigrantes. El racismo en el mundo obrero es poco ideologizado y se fundamenta, en primer lugar, en la experiencia de la competencia. Pétonnet (1968) ha mostrado que las categorías populares más precarizadas, a veces anteriormente inmigrantes, acusan claramente a los inmigrantes recien llegados de aprovecharse de los recursos del Estado de bienestar, cuyos beneficiarios deberían ser ellos mismos. Los temores y las competencias así como las solidaridades están presentes en las mentes. La figura del inmigrante atormenta el imaginario obrero en la medida en que revela la inseguridad de su estatus social. "Con todos los riesgos de incomprensión, de exclusión y de división que implica el descontrol de la situación: la clase obrera francesa es menos racista que la clase media de su país y mucho menos que otras clases obreras en Europa, pero se encuentra desprotegida ante el cambio"12.

La encuesta realizada en Sochaux por Beaud y Pialoux (1999) conduce a conclusiones relativamente pesimistas: la clase obrera vive un declive ineluctable de sus posiciones económicas, sociales y simbólicas. Lo vive como un drama y los discursos racistas recogidos a veces por los investigadores deben ser interpretados en este contexto, sobre todo cuando son recogidos por unos intelectuales antirracistas. La hostilidad de los obreros hacia todo lo que pudiera parecerse a la discriminación positiva a favor de los inmigrantes hace referencia al miedo que los azota de verse precipitados en una decadencia social insoportable para aquellos que, veinte años antes, pensaban conocer una promoción social. Más que la impresión de un racismo obrero, se puede considerar el voto obrero para el Frente Nacional como un último intento de diferenciación social y de reivindicación del derecho a existir en un contexto de desclasificación estructural de la clase obrera ${ }^{13}$. De Rudder y Vourc'h (2006), que se interesan por las dificultades sindicales en la lucha contra el racismo, consideran que estos no pueden seguir escondiendo el racismo existente en el ámbito laboral y empiezan a preocuparse por el que se expresa en su seno.

El trabajo histórico llevado a cabo por Noiriel (2007a) ofrece un amplio análisis de las relaciones entre la inmigración y el racismo, demostrando hasta qué punto este último utiliza las mismas vías desde la III República. Rechazando el vínculo entre el racismo y la colonización, insiste en el papel esencial desempeñado por los medios de comunicación, los expertos y los escritores en la construcción del discurso público. Este justifica las prácticas y las decisiones del poder y de la administración pública en materia de inmigración, convirtiéndolo en problema. Numerosos discursos aparentemente sabios defienden el "interés nacional" sugiriendo la selección de los inmigrantes.

\section{LOS EXTRANJEROS EN EL ESPACIO URBANO}

La ciudad es un lugar privilegiado para la escenificación de las diferencias. Los estudios sobre la vivienda han demostrado que los inmigrantes, a través de la articulación de su triple estatus de hombres, de obreros no cualificados y de extranjeros, son el objeto de mecanismos segregativos que tienden a concentrarlos en ciertos barrios y tipos de vivienda. En un primer tiempo, se ha impuesto la separación, ya que, durante mucho tiempo, han sido acantonados en centros $^{14}$, hoteles, chabolas y zonas de transito, antes de ocupar vi-

12 VERRET, M., L'espace ouvrier. Paris, Armand Colin, 1979, p. 168.

13 BEAUD, S.; PIALOUX, M., Retour sur la condition ouvrière. Paris, Fayard, 1999, p. 404-405.

14 BERNARDOT, M., “Chronique d'une institution: la Sonacotra 1956-1976”, Sociétés contemporaines, n 33-34, 1999. 
viendas ordinarias, sobre todo de protección oficial ${ }^{15}$. Contrariamente a la empresa, donde disponían de un estatus reconocido, la situación de los inmigrantes en el espacio local es lo que está en juego electoralmente. Son objeto de fantasmas y de estrategias de evitación. Estos espacios de convivencia han sido considerados como lugares de escenificación de las diferencias culturales. La ciudad es el lugar privilegiado de la exageración culturalista, a pesar de que sea imposible medirlo. Efectivamente, las poblaciones que han vivido la colonización están a menudo más aculturadas que las demás, lo que no significa que no sean víctimas de las discriminaciones. La sociología de la inmigración ha tenido que deconstruir estas representaciones para poner de manifiesto lo que esconden, sobre todo las estrategias de evitación.

Algunos trabajos académicos traducen la opinión común y persistente de que la inmigración constituye un problema. En 1974, surge una polémica entorno a la noción de "umbral de tolerancia" de la sociedad francesa hacia los extranjeros que una investigación del INED trata de cuantificar. Este umbral supone que, más allá de un cierto porcentaje de extranjeros en una zona geografica, los autóctonos manifiestan actitudes de rechazo. Las cuestiones planteadas a lo largo del sondeo de opinión aparecen extravagantes hoy en día para una investigación científica, pero demuestran el arraigo del binomio inmigraciónproblema. La connotación organicista de la noción de umbral, que presenta a los inmigrantes como si un cuerpo extranjero a la nación se tratara, está recusada por el conjunto de los investigadores que se dedican a la sociología de la inmigración, puesto que intentan precisamente deconstruir el sentido común y las prenociones que se esconden detras de numerosos discursos.

La trilogía realizada sobre tres barrios parisinos ${ }^{16}$ constituye una aplicación directa de esta deconstrucción. Transponiendo a Francia la tradición de Chicago, los autores articulan el espacio urbano, las posiciones sociales y las relaciones inter-étnicas. Así, los inmigrantes de los barrios favorecidos, el distrito n ${ }^{\circ} 16$ de París, no gozan de ninguna visibilidad. En el distrito $\mathrm{n}^{\circ} 13$, se constituye un barrio chino alrededor de comercios étnicos. El mercado de Aligre y el distrito $\mathrm{n}^{\circ} 12$ aparecen como unos lugares de coexistencia pacífica. Las reacciones de rechazo, de indiferencia, de solidaridad y de ayuda mutua se manifiestan siempre a propósito de parámetros económicos y simbólicos diferenciados. Las interacciones relacionan a unos actores distinguidos en función de la nacionalidad, el sexo, la edad, el estatus económico y la trayectoria tanto social como personal. Los conflictos no son considerados como desviaciones sino como unos procesos clásicos de aprovechamiento de las oportunidades. Si no se habla de tolerancia, el miedo al comunitarismo y al Islam refuerza el análisis culturalista. El ghetto ${ }^{17}$ es descrito en términos criminales y se considera como un enclave en la República. El estudio de la estratificación social y étnica del espacio se focaliza en los suburbios urbanos en los que se encuentran concentradas las poblaciones inmigrantes. Se le asocia una nueva figura: la de los jóvenes de los suburbios.

Esta situación conduce a los sociólogos a considerar que el espacio político local estructura las relaciones sociales. A través de un amplio trabajo de observación participativa llevada a cabo en un municipio obrero de la periferia parisina, Masclet (2003) pone de manifiesto tres procesos vinculados entre sí a la hora de comprender la crisis persistente de los suburbios. En primer lugar, insiste en el desconocimiento y la falta de reconocimiento por parte de las fuerzas políticas locales de un "nuevo proletariado" que se corresponde a la clase obrera clásica de los "barrios rojos". En segundo lugar, subraya el cierre del espacio político a todas las fuerzas de izquierda que no ofrecen ningún relevo real a los

15 BAROU, J., "Trajectoires résidentielles du bidonville au logement social", in DEWITTE, P., Immigration et intégration. L'état des savoirs. Paris, La Découverte, 1999.

16 TABOADA-LEONETTI, I., Les immigrés des beaux quartiers. Paris, CIEMI/L’Harmattan, 1987.

17 LAPEYRONNIE, D., Ghetto urbain. Paris, Robert Laffont, 2008. 
militantes asociativos provenientes de la inmigración. En tercer lugar, pone el énfasis en la derechización o la búsqueda de identificación comunitaria de una parte de la juventud descendiente de la inmigración decepcionada por la izquierda. Analizando las estrategias de los actores urbanos, tales como la evitación de los barrios estigmatizados, la investigación revela la discriminación en el acceso a la vivienda y el peso de las estrategias educativas que constituyen un vector central en el reparto de las desigualdades espaciales ${ }^{18}$.

\section{DESCALIFICACIÓN Y ETNICIZACIÓN DEL SISTEMA EDUCATIVO}

Los primeros en interesare por la escolarización de los hijos de inmigrantes han realizado un diagnóstico comparativo, enfrentándose al sentido común: el hijo de inmigrante que tiene otra cultura tendría dificultades por definición. En realidad, la pertenencia social sigue siendo preponderante en la medida en que el fracaso escolar es debido ante todo al origen mayoritariamente obrero de los descendientes de inmigrantes. Analizando la trayectoria escolar de los niños de los suburbios de la región de Sochaux en los años 1990, Beaud insiste en sus condiciones de socialización. Acumulan las carencias que difícilmente pueden ser segmentadas en variables separadas. El trabajo de campo pone de manifiesto lo que significa concretamente haber crecido en un suburbio durante los años de crisis: conocer las privaciones materiales y la angustia ante un futuro incierto, matricularse en escuelas e institutos situados en Zonas de Educación Prioritaria que resisten como pueden a la anomía ambiental, tener un horizonte geográfico que se limita al barrio, asistir a la decadencia de los hermanos mayores o de los vecinos, padecer el racismo y diversas formas de discriminación. Este pasado, que se cristaliza en el cuerpo y en la mente de los jóvenes, acaba por constituir una deficiencia inicial considerable en la competencia escolar y en la pugna para acceder a un empleo estable ${ }^{19}$.

El Instituto de Estadística francés, el INSEE ${ }^{20}$, propone un análisis de las relaciones que mantienen las familias con el sistema educativo. "A entorno social comparable, las familias inmigrantes se distinguen notablemente de las demás familias por su relación con el sistema educativo: expresan casi siempre aspiraciones escolares más elevadas". En este sentido, se produce una evolución de la problemática. Por una parte, las familias inmigrantes manifiestan su heterogeneidad, según los estatus sociales, las nacionalidades de origen y las zonas de escolarización. Por otra parte, con el transcurso del tiempo, las familias inmigrantes no mantienen la misma relación con el sistema educativo que al inicio de su estancia. El estudio se interesa por la movilización de las familias y su capacidad para aprovechar las oportunidades del sistema educativo. El interés se desplaza hacia la relación entre la escuela y su entorno. Un dispositivo de discriminación positiva territorializado se desarrolla en Francia al final de los años 1970: las Zonas de Educación Prioritaria (ZEP), que disponen de recursos humanos y materiales adicionales. Para lograr la clasificación en ZEP, se toma en consideración la presencia de niños inmigrantes que se asocia a un factor de fracaso escolar así como el comportamiento de las familias y las dificultades sociales.

Ya en la encuesta del INED de 1974, el niño inmigrante es presentado como un problema que justifica unas prácticas de evitación. Estudiando el fracaso escolar en un municipio del suburbio parisino, Léger y Tripier (1986) han demostrado de qué forma la supuesta ecuación "porcentaje elevado de niños inmigrantes = fracaso escolar de todos" estigmatiza el centro. Describen la existencia de una profecía auto-realizadora. Gracias al no respeto

18 OBERTI, M., L'Ecole dans la ville. Ségrégation, mixité, carte scolaire. Paris, Presses de Sciences Po, 2007.

19 BEAUD, S., 80\% au bac. Et après? Les enfants de la démocratisation. Paris, La Découverte, 2002, p. $304-305$.

20 CAILLE, P.; O'PREY, S., "Les familles immigrées et l'école française: un rapport singulier qui persiste même après un long séjour en France", Données sociales, 2003. 
del mapa escolar, la escuela de barrio obrero se convierte en un centro aún más popular, con más inmigrantes y con una tasa de fracaso escolar superior. La fama del centro escolar está cada día más vinculada al del barrio en el que se ubica. Esta situación resulta de un contexto de competencia entre los centros y del desarrollo de unas estrategias para preservar su prestigio. El conjunto de la vida social atestigua de la existencia de una frontera simbólica que tiende a desplazar las familias inmigrantes hacia el exterior de la comunidad educativa. Los estudios de Barthon (1997) y de Van Zanten (2002) han demostrado también de qué manera las estrategias de los diversos actores se entremezclan para escapar a la espiral de la descalificación: la preferencia de las familias por la escuela privada y las estrategias de reputación de los directores de centro. Este fenómeno se ha acentuado hasta el punto de hacer desaparecer el mapa escolar bajo el argumento de la necesidad de garantizar el derecho de las familias a la libre elección. Van Zanten (2006) habla de trivialización de la discriminación y hace suyo el análisis del círculo vicioso de la evitación que se fundamenta en la idea que la diversidad es un obstáculo para una buena escolaridad.

En su investigación sobre los colegios descalificados de la periferia de Lyon, Payet establece el proceso de etnicización de las relaciones educativas. Analizando la constitución de las clases de secundaria de un centro, pone de manifiesto los procesos de segregación interna al sistema educativo. La asociación "femenino-francés-precoz" constituye el polo valorizado del mundo escolar al que se opone el polo desvalorizado "masculino-magrebíen fracaso" $"$. Por su parte, Lorcerie (1999) muestra el deslizamiento progresivo de las problemáticas de estudio que empiezan con la etiqueta "hijos de inmigrantes", para orientarse poco a poco hacia la de "públicos difíciles", y acabar con la etnicidad. Demuestra que la referencia a la etnicidad va en aumento tanto en la escuela como fuera de ella, sin que los dispositivos de lucha contra las discriminaciones lo perciban ${ }^{22}$. La estigmatización étnica sirve para las estrategias de clasificación: quedarse entre iguales para los más favorecidos y evitar la desclasificación para los menos favorecidos. Si la etnicización de las relaciones sociales se desarrolla en todos los ámbitos de la sociedad, ¿qué ocurre en el sistema educativo considerado como el lugar por excelencia de la "indiferencia ante las diferencias"? El interés se centra en lo que sucede en el seno de la escuela, especialmente a propósito del velo islámico. Esta polémica ha relanzado el debate sobre la laicidad francesa y ha reafirmado la doxa republicana de la neutralidad de la escuela.

Félouzis y sus colaboradores han estudiado la segregación étnica en el colegio y sus consecuencias a partir del reparto de más de 144.000 alumnos de la academia de Burdeos. La construcción de la variable inmigrante se ha producido a partir del nombre de los alum$\operatorname{nos}^{23}$. Los resultados no son unívocos en términos de efectos sobre la calidad de la escolaridad aunque sean claros en lo que respecta a la segregación. "Las tasas de segregación son mucho más importantes en función del origen étnico que según el origen social o el retraso escolar" 24 . La atención se centra en los centros más segregados donde se plantea con una gran agudeza "la cuestión de la integración y de la igualdad de oportunidades". Perroton (2000), que ha participado en esta investigación, subraya la ambivalencia de esta etnicización de los alumnos así como de la escuela y de las familias. Las actitudes oscilan entre la valoración del prójimo y la estigmatización de las diferencias étnicas en razón de la norma verbal anti-racista y del peso de la tradición republicana. Uno de los componentes de la estigmatización de los niños descendientes de inmigrantes en el sistema educativo es la imputación de malos comportamientos. Durante el mismo periodo, la

21 PAYET, J-P., Collèges de banlieue. Paris, Méridiens-Klincksieck, 1995, p. 134.

22 LORCERIE, F., L'école et le défie. Paris, INRP/ESF, 2003.

23 FÉLOUZIS, G.; LIOT, F.; PERROTON, J., L'Apartheid scolaire: enquête sur la ségrégation ethnique dans les collèges. Paris, Seuil, 2005.

24 FÉLOUZIS, G., "La ségrégation ethnique au collège et ses conséquences", Revue française de Sociologie, vol. 44, n 3,2003 
asociación etnicidad y delincuencia se ha desarrollado como una evidencia ${ }^{25}$. El tema de la violencia y de las incivilidades está cada vez más vinculado a la juventud proveniente de la inmigración. El paso de la noción de dificultad o de conflicto al de violencia tiene como consecuencia que se resuelve un conflicto mientras que se reprime la violencia. A menudo, el conflicto tiene una dimensión colectiva y la violencia, además de referirse al individuo, oculta lo que está en juego.

\section{LOS DISTURBIOS URBANOS}

Los disturbios urbanos han sido el objeto de varias investigaciones ${ }^{26}$. Si la crisis del modelo republicano de integración de los jóvenes descendientes de inmigrantes es abordado a veces, la dimensión étnica de estos acontecimientos es frecuentemente eludida a favor de una interpretación que privilegia las causas sociales y espaciales. Bien es cierto que, en Francia, estos acontecimientos no han dado lugar a una movilización explícita fundamentada en la etnicidad. Las principales interpretaciones insisten especialmente en la crisis de los suburbios urbanos, la ausencia de movilidad social y las discriminaciones étnicas. En su libro, Bachmann y Le Guennec (1997) recuerdan la historia de las viviendas de protección oficial y de las grandes construcciones. Muestran de qué manera la esperanza despertada por estas construcciones durante los años 1960 y 1970 se ha transformado, con la ruptura del "ascensor social", en una crisis social aguda puesta de manifiesto por los disturbios.

Los trabajos de Beaud y Pialoux (2003) insisten en el aumento de la precariedad y de la desesperación social, la desestructuración del mundo obrero, de sus solidaridades y de sus organizaciones. Tras una investigación minuciosa, enuncian las causas de los disturbios: el abandono de estos barrios, la ausencia de un seguimiento de estos jóvenes por el sistema educativo y los servicios sociales, el fracaso escolar cada vez más costoso de los desfavorecidos, la aceptación de la precariedad estructural, la ausencia de solidaridad con esta juventud sacrificada, el racismo ordinario y la discriminación en la contratación alimentan la desesperación y el resentimiento creciente de estos jóvenes. Este análisis diverge un poco de la interpretación propuesta diez años antes por Jazouli (1986) y Dubet (1987), tras los rodeos de las Minguettes en 1981, y por Lapeyronnie (1993), después de los disturbios de Vaulx-en-Velin. Dubet considera que la acción colectiva llevada a cabo por los jóvenes marginados durante los disturbios resulta del racismo y, más precisamente, de las prácticas discriminatorios de la policía. Bachmann y Le Guennec (1997), por su parte, insisten en el factor que ha desencadenado estos disturbios, ya que se trata sistemáticamente de la muerte de un joven tras un enfrentamiento con la policía. El contencioso entre la policía y los jóvenes es permanente como lo atestiguan los estudios llevados a cabo tras los disturbios de 2005. Mucchielli y Le Goaziou (2006) demuestran que las prácticas policiales en estos barrios no constituyen una solución ante los problemas existentes sino un factor de enquistamiento. La conflictividad entre los jóvenes y la policía se prolonga incluso en el sistema judicial ${ }^{27}$. La estigmatización excesiva de la que son objeto estos jóvenes, especialmente en sus contactos con las fuerzas de seguridad y los constantes controles de identidad, hace que los perciban como unas provocaciones. Estas prácticas se perpetúan, lo que alimenta su odio de la policía.

Los disturbios de 2005 han permitido igualmente identificar las causas institucionales, tales como el menor compromiso del Estado con los dispositivos destinados a los subur- 
bios, lo que genera un sentimiento de abandono en estas zonas periféricas ${ }^{28}$. Esta implicación inferior se ha producido sobre todo en las actividades de animación, de renovación y de inserción. Lagrange y Oberti (2006) han analizado los efectos de las políticas locales sobre los disturbios, demostrando que si la acción pública local tiene poca incidencia sobre el inicio de los disturbios, puede reducir su amplitud. Lagrange considera que una actitud abierta en cuanto al reconocimiento de la pluralidad de las culturas de algunas municipalidades parece haber contenido los acontecimientos.

Si Beaud y Pialoux (2003) consideran que los disturbios son la consecuencia de una desesperación social, Dubet y Lapeyronnie (1992) insisten más en los sentimientos de desprecio y de injusticia sentidos por los jóvenes de estas zonas de relegación. Los disturbios están frecuentemente asociados a la rabia de los jóvenes excluidos que expresan una violencia sin proyecto. Lapeyronnie concede una dimensión política a los disturbios que define como el hecho de unos "primitivos de la protesta", es decir, la protesta de los que no tienen otra forma que la violencia colectiva para que les escuchen y entrar así en el espacio político. Esta interpretación es igualmente compartida por las investigaciones internacionales sobre los disturbios urbanos de los grupos étnicos minorizados. Según esta perspectiva, el disturbio como violencia colectiva no organizada es una manifestación política que no puede reducirse a la expresión de una criminalidad urbana. En su análisis sobre los argumentos utilizados durante los disturbios de 2005, Mauger (2006) formula una hipótesis similar hablando de protesta proto-política, es decir, de una protesta en contra de las prácticas estatales percibidas como ilegitimas. En este sentido, la protesta es una manera de entrar en el espacio político. No obstante, esta interpretación oculta ciertas prácticas de la sociabilidad de los suburbios, la efervescencia asociativa, la implicación política y la vitalidad de las prácticas solidarias ${ }^{29}$ que no consideran los suburbios como espacios completamente despolitizados ${ }^{30}$. Si los disturbios de 1983 conducen a la formación de una organización autónoma y a unas reivindicaciones políticas durante "la marcha por la igualdad", en 2005, la dimensión política es menor, mientras que las manifestaciones de violencia ponen el énfasis sobre las prácticas de unos jóvenes hombres que afirman su virilidad.

La dificultad de plantear la cuestión de la radicalización de las relaciones sociales en Francia conduce a infravalorar el desplazamiento de la cuestión social hacia la cuestión racial $^{31}$, aunque numerosas investigaciones subrayen el peso de la discriminación racial y de la segregación. Al contrario de los trabajos británicos sobre este tema, la cuestión del racismo político e institucional no se ha estudiado tanto. Sin embargo, esta temática es tratada como consecuencia de la aparicion del concepto de respeto que convierte los disturbios en acciones colectivas a favor del reconocimiento. La mayoría de los autores identifican de manera más explicita esta demanda de reconocimiento. Por último, algunos trabajos intentan determinar en qué medida las estructuras institucionales y políticas francesas contribuyen al advenimiento de los disturbios. Algunos ponen el énfasis en el cierre del sistema político galo y la poca disposición de las autoridades a reconocer organizaciones legítimas con el fin de negociar con ellas.

28 MUCCHIELLI, L.; LE GOAZIOU, V., Quand les banlieues brûlent. Paris, La Découverte, 2006.

29 KOKOREFF, M., La force des quartiers. De la délinquance à l'engagement politique. Paris, Payot, 2003.

30 KOKOREFF, M., "Sociologie de l'émeute. Les dimensions de l'action en question”, Déviance et société, vol. 30, n 4, 2006.

31 FASSIN, D.; FASSIN, E., De la question sociale à la question raciale. Paris, La Découverte, 2006. 


\section{CONCLUSIÓN}

Las investigaciones empíricas ven enfrentarse varias interpretaciones: la de las relaciones de clase, la de las relaciones de género y la de las relaciones interétnicas. El debate gira igualmente en torno al papel desempeñado por las instituciones que disponen de un poder de imposición superior al que goza la acción de las minorías dominadas. Demuestran que, para comprender unas situaciones concretas, conviene combinar ciertas variables y asociarlas en el tiempo. La aproximación entre las sociologías del género y del racismo es más evidente. Se desarrollan trabajos sobre las relaciones de género entre los jóvenes de los suburbios ${ }^{32}$. Refiriéndose a autores norteamericanos, varias investigaciones asocian la clase, el género y la etnicidad ${ }^{33}$. Por ejemplo, volviendo a la polémica sobre el velo islámico, que ha desembocado en la ley de 2004 sobre los signos religiosos ostensibles en el sistema educativo, Fassin relaciona el registro sexual y racial, puesto que la ley tiene que proteger a las víctimas.

\section{BIBLIOGRAFÍA}

AUBERT, F.; TRIPIER, M.; VOURC'H., F., Jeunes issus de l'immigration: de l'école à l'emploi. Paris, CIEMI/L'Harmattan, 1997.

AVENEL, C., Sociologie des quartiers sensibles. Paris, Armand Colin, 2005.

BAROU, J., "Trajectoires résidentielles du bidonville au logement social”, in DEWITTE, P., Immigration et intégration. L'état des savoirs. Paris, La Découverte, 1999.

BEAUD, S., 80\% au bac. Et après? Les enfants de la démocratisation. Paris, La Découverte, 2002.

BEAUD, S.; PIALOUX, M., Retour sur la condition ouvrière. Paris, Fayard, 1999.

BERNARDOT, M., “Chronique d'une institution: la Sonacotra 1956-1976”, Sociétés contemporaines, $\mathrm{n}^{\circ}$ 33-34, 1999.

CAILLE, P.; O'PREY, S., “Les familles immigrées et l'école française: un rapport singulier qui persiste même après un long séjour en France”, Données sociales, 2003.

DE RUDDER, V.; POIRET, C.; VOURC'H, F., L'inégalité raciste. Paris, PUF, 2000.

DE RUDDER, V.; VOURC'H, F.; TRIPIER, M., “Racisme et discrimination dans le travail: une réalité occultée”, L'Homme et la société, n 121-122, 1996.

FASSIN, D., "L'invention française de la discrimination", Revue française de science politique, vol. 52, $\mathrm{n}^{\circ} 4,2002$.

FASSIN, D., "Du déni à la dénégation. Psychologie politique de la représentation des discriminations", in FASSIN, D.; FASSIN, E., De la question sociale à la question raciale. Paris, La Découverte, 2006.

FASSIN, D.; FASSIN, E., De la question sociale à la question raciale. Paris, La Découverte, 2006.

FÉLOUZIS, G., "La ségrégation ethnique au collège et ses conséquences", Revue française de Sociologie, vol. $44, n^{\circ} 3,2003$

FÉLOUZIS, G.; LIOT, F.; PERROTON. J., L'Apartheid scolaire: enquête sur la ségrégation ethnique dans les collèges. Paris, Seuil, 2005.

HAMEL, C., "De la radicalisation du sexisme au sexisme identitaire", Migrations et Société, vol. 17, $\mathrm{n}^{\circ}$ $100,2005$.

KOKOREFF, M., La force des quartiers. De la délinquance à l'engagement politique. Paris, Payot, 2003.

32 HAMEL, C., "De la radicalisation du sexisme au sexisme identitaire", Migrations et Société, vol. 17, n 100, 2005.

33 POIRET, C., "Articuler les rapports de sexe, de classe et interethniques: quelques enseignements du débat nord-américain", Revue européenne des migrations internationales, vol. 21, n 1, 2005. 
KOKOREFF, M., "Sociologie de l'émeute. Les dimensions de l'action en question”, Déviance et société, vol. $30, n^{\circ} 4,2006$.

LAGRANGE, H.; OBERTI, M., Emeutes urbaines et protestations. Paris, Presses de Sciences Po, 2006.

LAPEYRONNIE, D., Ghetto urbain. Paris, Robert Laffont, 2008.

LORCERIE, F., L'école et le défie. Paris, INRP/ESF, 2003.

LORCERIE, F., "Le primordialisme français, ses voies, ses fièvres”, in SMOUTS, M.-C., La situation postcoloniale. Paris, Presses de Sciences Po, 2007.

MUCCHIELLI, L., Violences et insécurité. Paris, La Découverte, 2002.

MUCCHIELLI, L.; LE GOAZIOU, V., Quand les banlieues brûlent. Paris, La Découverte, 2006.

OBERTI, M., L’Ecole dans la ville. Ségrégation, mixité, carte scolaire. Paris, Presses de Sciences Po, 2007.

PAYET, J-P., Collèges de banlieue. Paris, Méridiens-Klincksieck, 1995.

POIRET, C., "Articuler les rapports de sexe, de classe et interethniques: quelques enseignements du débat nord-américain”, Revue européenne des migrations internationales, vol. 21, n 1, 2005.

PORTES, A., "Children of immigrants: segmented assimilation and its determinants", in PORTES, A., et al, The economic sociology of immigration. New York, Sage, 1995.

SANTELLI, E., La mobilité sociale dans l'immigration. Toulouse, Presses Universitaires du Mirail, 2001.

SAYAD, A., La double absence. Paris, Seuil, 1999.

TABOADA-LEONETTI, I., Les immigrés des beaux quartiers. Paris, CIEMI/L’Harmattan, 1987.

TAGUIEFF, P-A., Face au racisme. Paris, La Découverte, 1991.

VERRET, M., L'espace ouvrier. Paris, Armand Colin, 1979.

WIEVIORKA, M., La France raciste. Paris, Seuil, 1992.

WRENCH, J.; REA, A.; OUALI, N., Migrants, Ethnic, Minorities and the Labour Market. London, Macmillan, 1999. 